\title{
La expresión de la sexualidad durante la gestación y el puerperio
}

\section{The expression of sexuality during pregnancy and the postpartum period}

\section{A expressão da sexualidade durante a gravidez e o período pós-parto}

\author{
Sagrario Gómez Cantarino ${ }^{1}$; M. Moreno Preciado ${ }^{2}$ \\ ${ }^{1}$ Coordinadora de Enfermería Especialista del Complejo Hospitalario de Toledo. \\ Profesora Asociada UCLM (Campus Toledo. Enfermería \\ ${ }^{2}$ Profesor Titular. Universidad Europea de Madrid.
}

Cómo citar este artículo en edición digital: Gómez Cantarino S; Moreno Preciado M. (2012) La expresión de la sexualidad durante la gestación y el puerperio.Cultura de los Cuidados. (Edición digital) 16, 33. Disponible en: <http://dx.doi.org/10.7184/ cuid.2012.33.09>

Correspondencia: Sagrario Gómez Cantarino. Hospital Virgen de la Salud. Complejo Hospitalario de Toledo. Avda de Barber, 3045004 Toledo. Tel:: 925/269200. Extensión: 48559-48561.email: docenciamatronas@sescam.jccm.es Recibido: 03/12/2011/ Aceptado: 22/marzo/2012

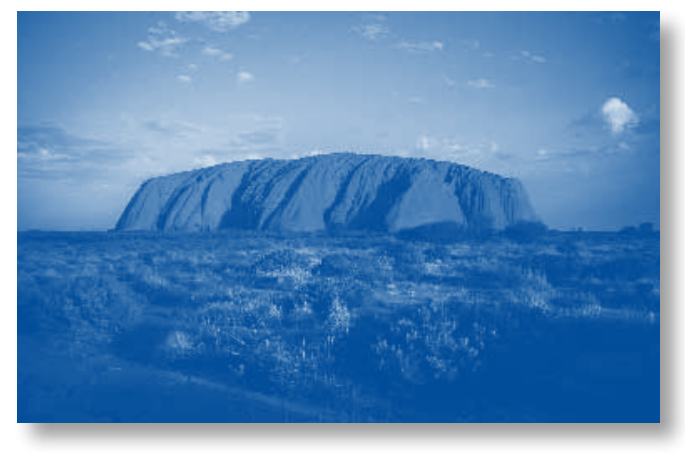

ABSTRACT

The mother concept, cannot be conceived as a fact isolated in time, without understanding the influences on women have had different events of history. The social, political and demographic, changes have conditioned the situation of women to evolve towards freedoms, rights, acquisition of new roles, occupation of positions in society that until now were only reserved for men. The nuptiality, fertility and birth rates have adapted to the new political and social circumstances. Motherhood carries a huge responsibility, means large demands almost exclusively to women from social, arriving to generate fear. The work of the health professionals involved in the care and counselling of the pregnant woman and puérpera will be offer care in a comprehensive manner, attitude of listening and support on issues related to sexual behaviour that requires an exquisite touch, as well as integrity, tolerance and understanding. Many women in this period requested help from medical personnel. Professionals dedicated to the care of the health of women, should influence of clear, explicit and systematically, to controls during gestation, in the exercise of sexuality as healthy behavior.

Key words: Sexuality, pregnant, midwife, health, puérpera.

\section{RESUMO}

O conceito de mãe, não pode ser concebida como um fato isolado no tempo, sem entender as influências sobre as mulheres tiveram diferentes acontecimentos da história. As mudan- 
ças sociais, políticas e demográficas, têm condicionado a situação das mulheres a evoluir no sentido de liberdades, direitos, aquisição de novas funções, a ocupação de posições na sociedade que até agora eram apenas reservados para os homens. O nuptiality, fertilidade e as taxas de natalidade adaptaram-se às novas circunstâncias políticas e sociais. A maternidade tem uma enorme responsabilidade, significa grandes demandas quase que exclusivamente para as mulheres da social, chegando a gerar medo. O trabalho da saúde profissionais envolvidos no cuidado e aconselhamento da mulher grávida e puérpera será oferecer cuidados de uma forma abrangente, a atitude de escuta e apoio em questões relacionadas ao comportamento sexual que requer um toque requintado, bem como a integridade, a tolerância e a compreensão. Muitas mulheres neste período solicitaram ajuda do pessoal médico. Profissionais dedicados ao cuidado da saúde das mulheres, deve influenciar de clara, explícita e sistematicamente, a controles durante a gestação, no exercício da sexualidade como comportamento saudável.

Palavras-chave: Sexualidade, grávida, puérpera, parteira, saúde.

\section{RESUMEN}

El concepto madre, no se puede concebir como un hecho aislado en el tiempo, sin comprender las influencias que sobre la mujer han tenido los diferentes acontecimientos de la historia. Los cambios sociales, políticos y demográfico, han condicionado que la situación de la mujer evolucione hacia libertades, derechos, adquisición de nuevos roles, ocupación de puestos en la sociedad que hasta ahora sólo estaban reservados para el hombre. La nupcialidad, fecundidad y natalidad se han adaptado a las nuevas circunstancias políticas y sociales. La maternidad conlleva una responsabilidad gigantesca, implica grandes exigencias impuestas casi exclusivamente a la mujer desde lo social, que llegan a generar miedo.

La labor de los profesionales sanitarios que participan en el cuidado y asesoramiento de la gestante y puérpera será ofrecerles una atención de forma integral, actitud de escucha y de apoyo sobre cuestiones relativas al comportamiento sexual que exige un tacto exquisito, así como integridad, tolerancia y comprensión. Muchas mujeres en este periodo solicitan ayuda del personal sanitario. Los profesionales dedicados al cuidado de la salud de la mujer, deberían incidir de forma clara, explícita y sistemática, al hacer los controles durante toda la gestación, en el ejercicio de la sexualidad como conducta saludable.

Palabras clave: Sexualidad, gestante, matrona, sanitario, puérpera.

\section{INTRODUCCIÓN}

La salud sexual y reproductora, es el estado de bienestar en torno a los hechos relacionados con la reproducción y la sexualidad humana. Abarca conceptos de salud, entendida es su dimensión histórica, cultural, social e individual. La salud reproductora entraña la capacidad de disfrutar de una vida sexual satisfactoria y sin riesgos, y de procrear, y la libertad para hacerlo o no hacerlo, cuándo y con qué frecuencia. La sexualidad recorre de forma transversal la vida de todo ser humano, desde que nace hasta que muere. Más aún, le conforma como persona y marca su identidad, y por tanto su subjetividad, su forma de percibir el mundo, de sentir y de actuar. (Andrés Domingo A, 2000). La reproducción es un hecho individual, se produce 
en personas concretas con relaciones particulares, pero es también un hecho social y cultural, ya que las personas viven en estructuras sociales específicas y cumplen o transgreden mandatos sociales bajo unos valores y normas que conforman el universo de sus creencias. (Sanz F, 1992).

La reproducción es un hecho histórico, es cultural, diferente en el tiempo según el tipo de sociedad en que se ubique, sustentado por diferentes creencias religioso-culturales, por los diversos valores y normas sociales reguladoras, que afectan e influyen de manera distinta sobre hombres y mujeres, sobre adolescentes o adultos, sobre ricos o pobres, etc. La reproducción es un hecho sexual en la especie humana, que abarca diferentes actividades en torno al coito, que es el medio idóneo para conseguir el fin inmediato, el embarazo, como primer paso necesario para la reproducción de los individuos y de la especie. La realidad es, que nuestra cultura y nuestra sociedad es coitocéntrica, lo que significa la sobrevaloración de esta actividad sexual, como la máxima expresión de la sexualidad, como la plenitud, (Fernández de Quero, 1996; OMS, 1978).

En España el tema de la sexualidad sufrió un proceso de apertura en la época de la transición (años 1975 a 1978), tras una larga dictadura, el espíritu de progreso y apertura inició un lento cambio en la mentalidad de la población. Debemos tener en cuenta que la población española y su concepción de la sexualidad sufrieron un profundo cambio en los últimos 20 años. La generalización del uso de los métodos anticonceptivos, el cambio en las prácticas sexuales, las enfermedades de transmisión sexual y las variaciones en conceptos de sexualidad, han hecho posible estos cambios de pensamiento, y esto también ha ocurrido durante el embarazo.
La Enfermería, como disciplina especializada en el campo de los cuidados, asume una gran responsabilidad en el fomento, protección y promoción de la salud sexual. Su intervención en esta área de la salud humana tiene por objetivo la atención integral de la persona para conseguir una buena calidad de vida. Las intervenciones de Enfermería se justifica si se tiene presente la necesidad de cuidados para el fomento de la salud sexual. La importancia de la prevalencia de enfermedades relacionadas directamente con los estilos de vida sexual exige programas de educación e intervención comunitaria con una participación directa y activa de la enfermería.

La sexualidad siempre ha estado impregnada de misterio, oscurecida por razones varias, encarcelada en la ignorancia y objeto de humor compartido en gran medida por el mismo sexo. La sexualidad como expresión de la personalidad, es única, personalizada e irrepetible, llegando a ser un sello personal de cada pareja y por tanto cuando se enfrenta el proceso reproductivo (gestación) debe existir un proceso de aceptación y adaptación a él, y a la variación del disfrute sexual en la medida de las posibilidades y deseos. Tanto el embarazo como la sexualidad han sido considerados tabúes. Hasta hace poco, los gine-obstetras guardaban silencio al respecto y la pareja se sentía intimidada para abordar el tema con el médico, inclusive entre ellos mismos. (González Labrador, Miyar Pielga; 2001).

Durante la gestación es factible que las parejas experimenten alteraciones en sus patrones sexuales, y esto muchas veces, se debe a la existencia de creencias erróneas en relación a la sexualidad. (Luz Marina Alonso y cols; 2003). La falta de educación, al igual que creencias erróneas, lleva a la alteración de los patrones sexuales entre los cónyuges, los cua- 


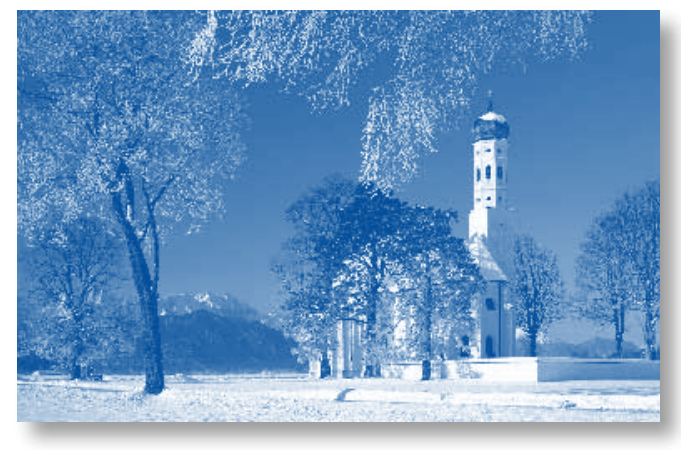

les pueden tener resultados adversos en la relación de pareja. En el momento en que se superen todos los temores y creencias erróneas, lo que se logra con una buena comunicación entre el personal de salud y la pareja, ésta última expresando cada uno sus sentimientos y dando grandes dosis de cariño, complicidad, paciencia, caricias y palabras, se podrá lograr disfrutar nuevas formas de satisfacción sexual distintas del coito y orgasmo, aprovechando un elemento positivo de esta época como es la libertad que siente la pareja al no tener que preocuparse por utilizar métodos anticonceptivos.

\section{OBJETIVOS}

\section{- OBJETIVO GENERAL:}

Obtener una mejor comprensión de los factores que intervienen en la vivencia de las mujeres gestantes y puérperas, respecto a como expresan su sexualidad durante su gestación y puerperio, así como de los profesionales que proporcionan asesoramiento e información al respecto. Mi intención es describir, traducir, explicar e interpretar el fenómeno, y en la perspectiva de que este análisis contribuye al fomento y disfrute de la sexualidad.

\section{- OBJETIVOS ESPECÍFICOS:}

\section{Acerca de la mujer:}

- Conocer las vivencias sexuales de las mujeres gestantes durante la gestación.
- Analizar las relaciones que se establecen durante las charlas de educación maternal entre las mujeres gestantes/matronas.

- Analizar tabúes, moralidad, creencias, ideologías, actitudes y valores que se manifiestan en esta etapa de su vida.

\section{Acerca de la matrona:}

- Conocer y analizar los significados, ideas y valores respecto a la sexualidad durante la gestación.

- Analizar las interacciones que se establecen durante la educación maternal, así como en los talleres de apoyo entre las mujeres asistes a las mismas.

\section{METODOLOGÍA}

- La metodología utilizada en esta investigación es la cualitativa asociada a estudios antropológicos como acontecimiento cultural, no sólo biológico, y desde el relato de la experiencia de las propias actoras. Dentro de este estudio he pretendido conocer, para luego analizar e interpretar los sucesos que rodean a la mujer sobre la expresión de su sexualidad durante la gestación y puerperio, así como a los sujetos tanto a nivel social como profesional en él implicados, que reflejen la trayectoria cultural de la socialización tanto de la mujer como de la expresión de su sexualidad.

- Teniendo acceso al relato de primera mano de las mujeres y profesionales, de cómo se interpreta y percibe el proceso de gestación y sexualidad, así como sus ideales respecto a esto, unido a cómo lo experimentan y las experiencias que llevan a cabo.

- Comenzar una etnografía es también abrir a la vez un diario de campo, ya que es el instrumento de registro fundamental del procedimiento de investigación en el que se inscriben paso a paso y desde los primeros 
momentos del proyecto, las actividades del etnógrafo.

- Las técnicas principales utilizadas para la recogida de la información, han sido la entrevista en profundidad y la observación participante.

- La recogida de datos se llevó a cabo entre mayo del 2009 y diciembre de 2010.

\section{DESARROLLO}

Se denota como las mujeres pertenecientes al estudio adoptan un papel social ya estipulado y marcado culturalmente, donde las normas morales de comportamiento para la mujer así como los prejuicios, continúan existiendo, siendo expresados por parte de estas. Cabe destacar como en estas mujeres, aún quedan estigmas socios culturales de toda la socialización vivida por las madres de las gestantes, que en realidad han sido las encargadas de formar y educar a las gestantes entrevistadas. Las mujeres jóvenes, expresan abiertamente sentir vergüenza cuando se habla de sexualidad, sexo, y todo lo que concierne a este tema con sus madres a nivel privado; huyendo generalmente de iniciar este tema, tanto por parte de la madre como por la hija. Por ejemplo entre estas mujeres está muy extendido el relacionarse y entablar temas sexuales con sus coetánea más cercanas, siendo estas amigas, amigos y compañeros de escuela, instituto e incluso de universidad, siendo esta información en un principio precarias y aumentando el nivel de las mismas según aumenta la edad de las mujeres como de las personas que la rodean. Se valora que una vez que se encuentran embarazadas, la información sobre temas sexuales la reciben por parte de la figura de la mujer, siendo esta generalmente información no científica y sin razonamiento en el que apoyar la información que ofrecen. Variando sustancialmente esta cuestión debido a la presencia de profesional sanitario en la zona de estudio, que complementa, aclara e instruye a las mujeres acerca de temas sexuales sin obsesiones ni tapujo al respecto. Por el contrario a nivel público, durante el desarrollo de los talleres de educación para la salud, se denota que este "tabú" existente y esta vergüenza presente entre madre e hija, se diluye, de tal manera que se comentan abiertamente situaciones referentes al sexo y la sexualidad por parte de las madres así como de las hijas, siendo esto más acuciado en la zona rural que en urbana; produciéndose esto debido a que el acompañamiento de la gestante y puérpera al centro de salud se realiza en gran medida con la madre y no con la pareja dentro de la zona rural. Se denota que el inicio de una relación estable entre un hombre y una mujer, hasta consolidarse como pareja, lleva implícito en sus inicios el esconderse, el verse de manera aislada, evitando así "el que dirán". Cabe destacar que esta situación se encuentra más enraizada dentro de la zona de estudio, cuando la mujer ha tenido más de una pareja; situación vivida y mal vista por sus coetáneos. Se valora como son las mismas mujeres las que comentan sobre estas el ser "algo fresca" e incluso "ligera de cascos", situación que no se ha dado y mucho menos ha sido expresado por las mujeres hacia la figura del hombre, aunque a este se la hayan conocido varias parejas. Los prejuicios sociales están muy marcados tanto en zona urbana como rural. Se evidencia como las mismas mujeres son las que exteriorizan que "esconden" amores, sentimientos, gestaciones prohibidas por el hecho de no estar bien visto por su entorno social. Todo esto es debido a que hasta hace pocos años la ideología católica existente reforzó el ideal de que la mujer debía ser casta, pura y sobre todo formal. Aún así las 
prácticas sexuales de cada pareja son únicas e irrepetibles, y las vivencias actuales son "contadas externamente", extrapoladas al exterior sin tapujos, buscando salidas, ayuda, incluso diría que sincerarse con ellas mismas. Cabe destacar que lo que más importa no es tanto saber qué es la mujer, como funciona el cuerpo femenino, en qué consiste la sexualidad femenina, cuáles son los valores propios de una cultura femenina que habría que perpetuar, desarrollar o crear, sino saber entender cómo se organiza la diferencia sexual en la cultura. Se debe tener en cuenta que no existen recetas mágicas, a la hora de poner en práctica la sexualidad durante la gestación y puerperio, ya que cada mujer es única, y cada pareja actúa de forma diferente ante una misma situación, y por lo tanto únicas son también sus experiencias. Socialmente la familia, los amigos y allegados dan consejos y recomendaciones de todo tipo, sobre todo entre mujeres aproximadamente de la misma edad, influyendo todo esto en la decisión personal de la mujer y de pareja. Así se dejan de mantener relaciones sexuales durante la gestación debido a la información recibida, sin ningún motivo "real", solo por el hecho de "prohibirlo", sin llegar a pensar que cada pareja y cada persona tienen necesidades diferentes y viven situaciones diversas, muchas se asemejan pero no son iguales, así lo comentan ellas mismas. En este paso es muy importante la buena autoestima, la capacidad de poner límites y la toma de decisión-acción por parte de la gestante, puérpera e incluso del profesional sanitario encargado de formar e informar a estas mujeres. En definitiva, se denota que parte de las mujeres de la zona de estudio valoran como la liberación de la mujer y la educación de la misma ha contribuido a que ésta obtenga mayor placer y satisfacción en sus relaciones, ya no solo destinadas para

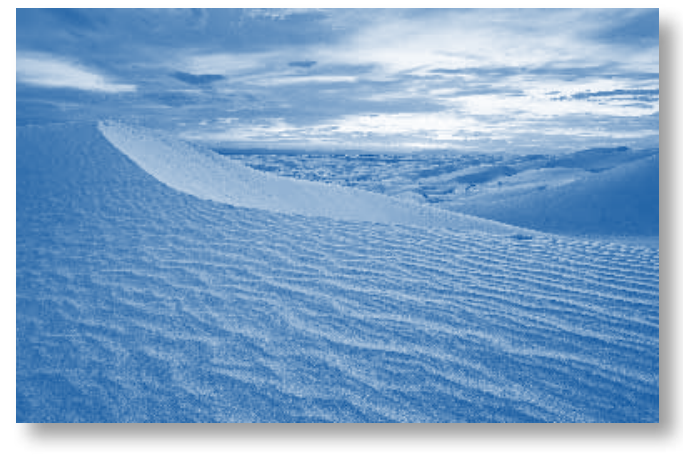

la reproducción. Se aprecia que la sociedad en la que conviven tanto mujeres como hombres continúa influyendo sobre la conducta sexual de estos; se valora que se está consiguiendo eliminar falsos mitos y tabúes que rodeaban el acto sexual, en gran medida debido a las charlas de profesionales, así como a las reuniones que realizan las propias mujeres en el centro de salud. Según he podido recoger en testimonios de las mismas mujeres, se necesita tomar mayor conciencia para tratar libremente todos los aspectos de la sexualidad, sin culpabilidad, vergüenza o pudor. Se puede comprobar que la mujer como su pareja se implica en la maternidad, en las relaciones sexuales y en compartir espacios, esto tan simple, para sus madres era impensable. Algunos de los hombres presentes en las charlas expresaron sentirse muy implicados en el desarrollo de la gestación, siendo esto más marcado en zona urbana. Se denota la sensibilidad como la sincronía, estimulación, así como la actitud positiva ante la nueva situación y la implicación de ambos en este nuevo momento que les corresponde vivir a ambos, sin tapujos, sin vergüenza y sin prohibiciones en un momento de cambio tanto fisiológico como psicológico para la mujer y su pareja. Las matronas entrevistadas previamente han realizado su función asistencial dentro de la Atención Especializada, coincidiendo en afirmar que existe un gran clima de equipo dentro de los centros de salud donde desarro- 
llan su función asistencial, no solo con la enfermería, si no, con todos los componentes del equipo multidisciplinar. Todas ellas expresan estar muy satisfechas dentro de la Atención Primaria de salud, no teniendo intención de trasladarse a la Atención Especializada. Dentro del centro de salud urbano Lisa, pude observar como los trabajadores y la población de dicho centro mantiene un trato estrecho, conociéndose entre ellos. En el centro de salud urbano la Luz, profesionales/usuarios no parece conocerse tan a fondo, pero debo señalar que en este centro es el propio equipo multidisciplinar quien gestiona los recursos humanos y materiales; en el centro de salud rural de la Patrona, tanto personal como comunidad se conocen manteniendo incluso lazos estrechos de amistad entre algunos de ellos. Incluso los usuarios relacionan centro de salud con la compra diaria, (la frutería queda enfrente del centro de salud, por lo que la mayor parte de mujeres acuden al centro con bolsas llenas de frutas). Estas matronas tienen estipulado un tiempo en la consulta programada, de unos quince minutos, siendo escasas para algunas de las mujeres que asisten a estas consultas, no existen quejas de las mujeres en ninguno de los centros donde se desarrolló el estudio por la espera realizada.

El equipo multiprofesional dentro de AP, del centro de salud, interactúa conjuntamente, en la atención a la mujer gestante y puérpera. Es un sistema de trabajo que permite a varias personas de diferentes profesiones y habilidades coordinar sus actividades, y en el cual cada uno contribuye con sus conocimientos al logro de un propósito común, en este caso el beneficio de la mujer. En ocasiones estos equipos programan charlas conjuntas, e incluso puestas en común de experiencias mutuas. Las matronas de los centros de salud donde realicé el estudio, tienen definidas sus funciones o roles, uno enmarcado en el modelo biomédico, consistente en realización de pruebas como curas y las técnicas contempladas en el programa de atención a la mujer gestante, este programa debe de cumplirse estrictamente. La segunda función queda enmarcada en su propio perfil como cuidadoras, y cercanas a la mujer, muy reconocido por la propia mujer. Realizan la atención a esta dentro de la consulta programada, (dando lugar esto ha realizar otro tipo de tareas y actividades dentro de su horario de trabajo), y la consulta a demanda en la cual la mujer acude por iniciativa propia. La historia obstétrica la inicia la matrona en la primera consulta de la mujer, en la captación de la gestante, esta historia obstétrica se utiliza tanto en AP como AE, por parte de los profesionales que valoran a la mujer, ya sea médico, enfermera, matrona, etc.

Respecto a la observación realizada en dichos centros de salud, pude comprobar como la educación sanitaria que imparten las matronas así como profesionales del equipo multidisciplinar, no es impartida ni recibida de la misma forma por parte de los profesionales, dependiendo de la ubicación del centro (zona urbano, como rural), así como de la formación de cada profesional sanitario, la visión respecto a la educación sexual de la mujer varía, realizándose esta actividad de manera diferente. Se observa que el léxico utilizado por los profesionales varía dependiendo de la formación del grupo de gestantes, según expresan los propios profesionales es importante conocer las características de su población, sobre todo cuando se trata de educación sexual, ya que valoran qu esta información dependiendo del grupo de mujeres a educar y formar se dará a nivel individual o grupal. Así en la zona urbana observé como la educación sexual en mayor 
medida se da a nivel individual, aunque también se imparte a nivel grupal, teniendo una mínima participación por parte de la mujer. Mientras que en la zona rural, esta formación la reciben las mujeres y acompañantes tanto a nivel individual como grupal.

\section{RESULTADOS}

Los resultados que presento, han sido obtenidos mediante el cruce de dos puntos de vista, uno -emic- de los actores e informantes, y otro -etic- perteneciente a mis categorías conceptuales. Fruto de este intercambio surgieron en el campo aspectos significativos no previstos inicialmente y que constituyen las categorías emergentes.

He realizado el análisis de los datos en torno a tres niveles: 1. Elaboración de categorías de análisis. 2. Agrupación en categorías más amplias. 3. Identificación de dominios cualitativos. Estos tres dominios teóricos es sobre los cuales trataré de explicar los resultados del estudio y los nombraré: percepción de la mujer, percepción de los profesionales, y percepción de las mujeres como usuarias del servicio de salud de Castilla- La Mancha.

\section{CONCLUSIONES}

El embarazo, es vivido de manera distinta por hombres y mujeres, puede afectar la vida y la sexualidad de diversas maneras según el sexo. La sexualidad como expresión de la personalidad, es única, personalizada e irrepetible, debe existir un paso de aceptación y adaptación a él y a la variación del disfrute sexual en la medida de las posibilidades y deseos. La nueva formación de enfermería especialista en obstetricia y ginecología, se contempla vía EIR (enfermero interno residente), cursándose por un periodo de dos años naturales y en la cual se contempla tanto la obstetricia como la gine- cología, incorporándose la formación en cuidados sexuales de la mujer y familia, como a la educación sexual en todas las etapas de la vida de la mujer. La educación sexual se hace cada vez más necesaria en una sociedad que parece estar muy bien informada. Sin embargo, a juzgar por la observación realizada así como en el desarrollo de las entrevistas, se conciben altas dosis de desconocimiento y errores, tanto en mujeres jóvenes como en adultas, que afecta gravemente al equilibrio emocional. Enseñar a conocer y aceptar el propio cuerpo, a buscar información o a demandar ayuda es educar para ser capaces de establecer con los demás unas relaciones más sanas y satisfactorias.

\section{BIBLIOGRAFÍA}

- Alonso L.M., Pérez M.A., Arias C., Figueroa N., Gamarra C., Martínez A., et al, (2003). Características biopsicosociales y frecuencia de relaciones sexuales de las embarazadas en la ESE Prudencio Padilla Clínica Sur. Colombia: Barranquilla.,(7-10).

- Andrés Domingo A. (2000). Salud sexual y reproductora. Salud Pública y Enfermería Comunitaria. 2a ed. Madrid. Barcelona, 1461-1490.

- Fernández de Quero, J. (1996). Guía práctica de la sexualidad masculina. Ediciones Temas de hoy. Madrid.

- González Labrador, I. y Miyar Pielga E. (2001).Sexualidad femenina durante la gestación. Revista cubana de Medicina General Integral. 17(5), 497-501.

- Orden SAS/1349/2009, de 6 de mayo, por la que se aprueba y publica el programa formativo de la especialidad de Enfermería Obstétrico-Ginecológica (Matrona).

- Sanz, F. (1992). Psicoerotismo masculino y femenino. Barcelona: Kairos 